\title{
Bilateral Pleural Effusion and Pulmonary Edema Caused by a Fistulous Aneurysm of the Ductus Arteriosus
}

\author{
Tetsuo Noguchi*, Kunihiko KamaKari*, Nobuhiro Ono*, Masaya Murata, Mitsuru Kitano and Yasuji Terada*
}

\begin{abstract}
We report an unusual case of pulmonary edema and rapid collection of bilateral pleural effusion caused by a fistulous large aneurysm of the ductus arteriosus (DAA). The diagnosis was performed by contrast $\mathrm{CT}$ and aortography. The cause of pulmonary edema and effusion was thought to be both elevated capillary pressure due to overload of the pulmonary circulation and decreased water clearance due to compression of the lymphatic system by the large DAA itself. Therefore, fistulous DAA should be considered when a continuous heart murmur and swelling in the aortic window are recognized. Once DAA is diagnosed, surgery should be performed without delay.

(Internal Medicine 41: 1029-1031, 2002)
\end{abstract}

Key words: aneurysm of the ductus arteriosus, pulmonary edema, pleural effusion

\section{Introduction}

Aneurysm of the ductus arteriosus (DAA) in adults is rare and no previous case of respiratory failure from pulmonary edema and effusion caused by DAA has been reported. We present an adult case of fistulous large DAA compressing the pulmonary artery and bronchus. The patient showed acute respiratory failure from pulmonary edema and effusion. His symptoms disappeared following a successful surgical aneurysmectomy using a Dacron patch.

\section{Case Report}

A 70-year-old man was admitted to our hospital with cough and increasing dyspnea. He had had an uneventful childhood with no significant medical history. On examination, moist rales in both lungs and a grade III/VI machinery murmur in the left third intercostal space were audible. Blood pressure of 104/50 $\mathrm{mmHg}$ and a regular pulse rate of 108 beats per minute were noted. An electrocardiogram showed no ischemic change and no arrythmia except tachycardia. Chest X-ray examination showed an infiltration shadow and effusion on both sides and swelling in the left upper mediastinum (Fig. 1 left). The mediastinal swelling had been pointed out to the patient 6 months earlier at another clinic, but he had ignored it. Arterial blood gas analysis showed $\mathrm{PaO}_{2} 48.6 \mathrm{mmHg}, \mathrm{PaCO}_{2} 35.8 \mathrm{mmHg}$, and $\mathrm{SaO}_{2} 83.3 \%$ on room air. CRP was $13.4 \mathrm{mg} / \mathrm{dl}$ and antibiotic (IPM/CS) was started. Two days later, the patient was intubated because of acute respiratory failure with deterioration of pulmonary edema and rapid accumulation of effusion (Fig. 1 right). A trocar catheter was inserted into each pleural space and 500-1,000 $\mathrm{ml}$ transudate pleural effusion (protein $1.0 \mathrm{~g} / \mathrm{dl}$, LDH $63 \mathrm{IU} / l$ ) was drained every day. After intubation, echocardiography was performed but no abnormal movement of the cardiac valves and ventricles was recognized. With respirator control and effusion drainage, the patient's arterial blood gases gradually improved and he was extubated 9 days after admission. Contrast-enhanced CT of the chest performed on the following day revealed bilateral residual pulmonary edema and a vascular mass in the aortic window pressing upon the main pulmonary artery and left main bronchus (Fig. 2). Right heart catheterization demonstrated moderate pulmonary hypertension (mean pulmonary artery pressure $30 \mathrm{mmHg}$ ) and a marked increase in the oxygen content from the right ventricle $\left(\mathrm{O}_{2}\right.$ CT $\left.12.0 \mathrm{vol} \%\right)$ to the main pulmonary artery $\left(\mathrm{O}_{2} \mathrm{CT}\right.$ $18.9 \mathrm{vol} \%)$. Qp/Qs was calculated as 4.18 . The ejection fraction rate was $73.1 \%$ and cardiac output was $14.0 \mathrm{l} / \mathrm{min}$ (hyperdynamic status). Three-dimensional CT scan and aortography (Fig. 3) confirmed that the mass was a fistulous DAA.

Soon after extubation, pulmonary hemorrhage occurred, and an emergency operation was performed through a left anterolateral thoracotomy. Following a partial cardiopulmonary bypass, the aorta was clamped proximally between the left carotid and left subclavian arteries and distal to the aneurysm. The DAA measured $12 \times 11 \mathrm{~cm}$. After incision of the aneurysm and evacuation of mural thrombus and atheroma, the pulmonary and aortic ends of the DAA were closed using a Dacron patch. The patient was then weaned uneventfully from the partial cardiopulmonary bypass. After the operation, the pulmonary edema and effusion disappeared. The postoperative course was uneventful and the patient was discharged 27 days after

From *the Department of Pulmonology and the Department of Cardiovascular Surgery, Nagahama City Hospital, Nagahama

Received for publication April 19, 2002; Accepted for publication August 9, 2002

Reprint requests should be addressed to Dr. Tetsuo Noguchi, the Department of Pulmonology, Nagahama City Hospital, 313 Oinui-cho, Nagahama 526-8580 

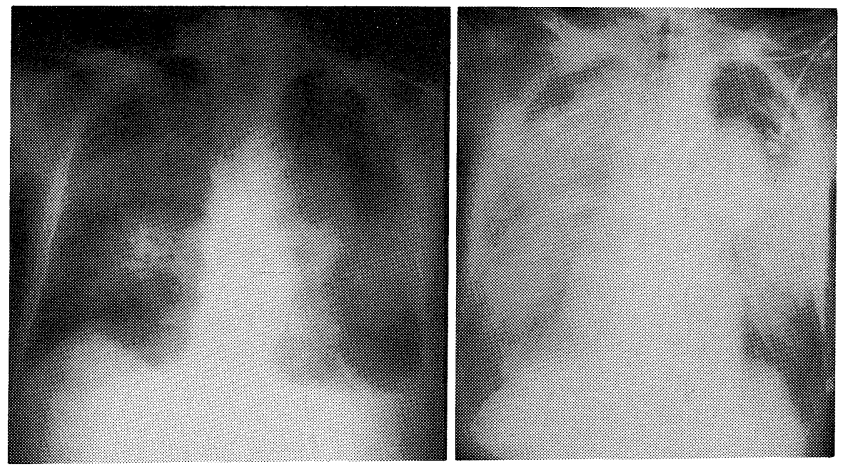

Figure 1. Infiltration shadow and bilateral effusion are evident on the chest $X$-ray film taken on admission (left). Two days later, deterioration of pulmonary edema and rapid accumulation of effusion occurred (right).

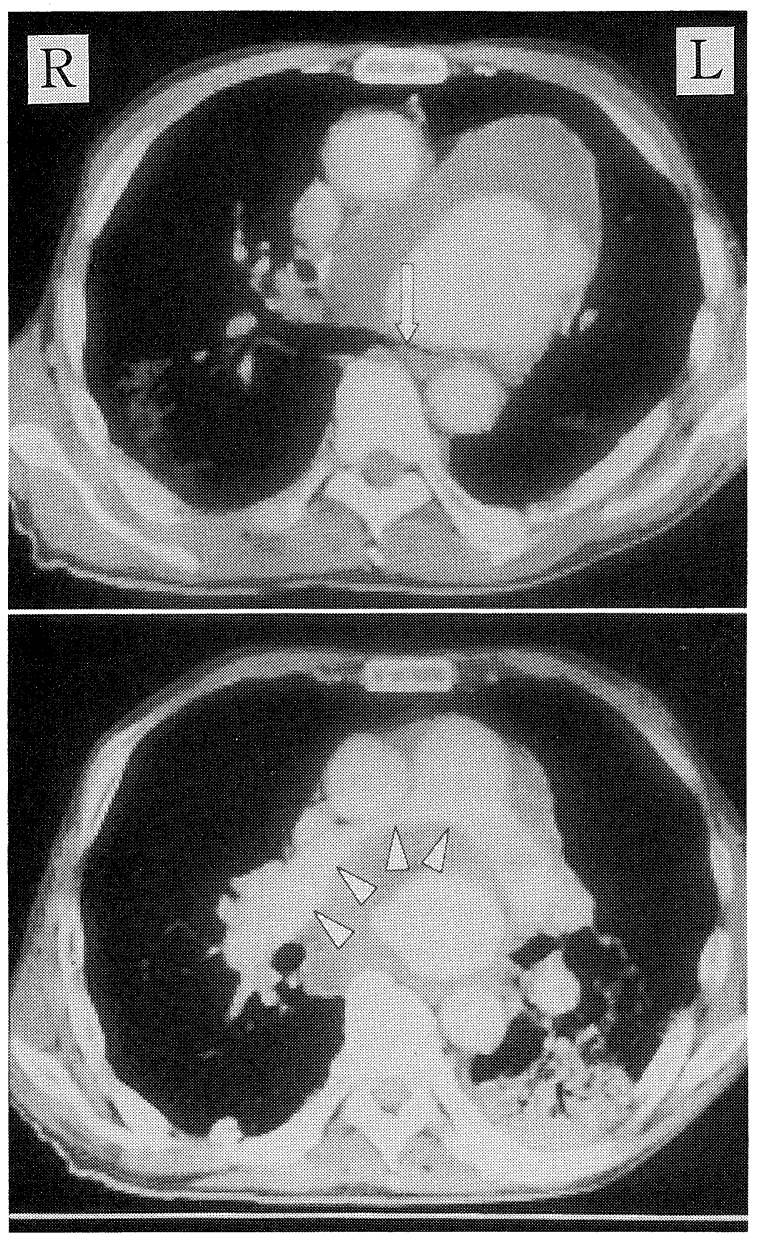

Figure 2. A large DAA with mural thrombosis is noted in the aortic window with compression of the left main bronchus (arrow; top) and main pulmonary artery (arrowheads; bottom).

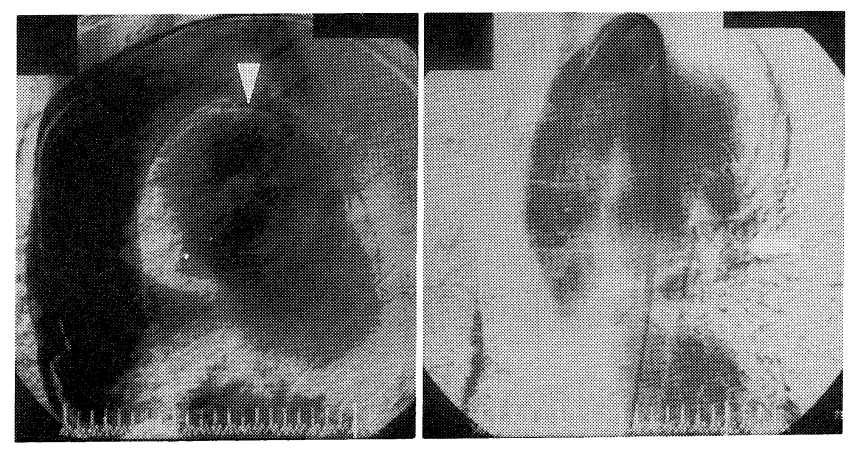

Figure 3. The aneurysm was derived from the aortic arch (arrowhead; orifice) and extended to the left pulmonary artery (left). The left pulmonary artery (arrow) was enhanced just after the aortic enhancement (right).

surgery.

\section{Discussion}

The mechanism of gradually developing DAA in adults remains uncertain. A leading theory has been proposed by Taussig, who reported that DAA is derived from delayed closure of the aortic end of the ductus arteriosus (1). Normally, closure of the ductus arteriosus begins at the pulmonary end, progressing towards the aorta. If closure of the aortic end is delayed, the weakened wall of the ductus will be exposed to systemic pressure which may lead to aneurysm formation (1). Although in most of the adult cases the ductus arteriosus was closed at the pulmonary end; only 5 cases of patent ductus at the pulmonary end have been reported previously (2-6). Approximately twothirds of the patients were long-term hypertensives (7), although the present patient had normal blood pressure.

Specific symptoms of DAA are few in adults, but the aneurysm may cause hoarseness, cough, anorexia and thoracic pain due to local effects on adjacent organs and nerves (8). When the DAA is patent at the pulmonary end, a continuous murmur may be audible, and dyspnea and peripheral edema will be dominant due to pulmonary hypertension and overload from the large volume of blood shunted from the systemic to the pulmonary circulation (8). Serious complications following spontaneous DAA are rupture, erosion into the adjacent organs (bronchi, esophagus), infection (arteritis, endocarditis) and thromboembolism. Recently, there has been an increase in the reported incidence of these events which may be largely due to improved diagnostic methods. Contrast-enhanced CT $(9,10)$ and aortography are essential for making a diagnosis. In neonates, transthoracic echocardiography has shown convincing potential, whereas in older children and adults, transesophageal echocardiography has yielded very promising results (8).

In the present patient, rapid accumulation of bilateral pleural effusion along with pulmonary edema occurred. We first thought that the pulmonary edema was cardiogenic such as an 


\section{A Large DAA Can Cause Pulmonary Edema}

aortic valve disorder caused by aortic aneurysm from the continuous murmur and chest $\mathrm{X}$-ray, but there were no abnormal findings upon echocardiography. Therefore, the diagnosis was not made until CT was performed after extubation.

To our knowledge, there has been no reported case of pulmonary edema and rapid effusion requiring drainage with fistulous DAA. We think the reason that our patient developed pleural effusion was because the pulmonary circulation increased rapidly due to the large fistulous DAA; the visceral pleural capillary pressure was also elevated, which resulted in a higher inflow volume into the pleural space. Furthermore, the large DAA (diameter $12 \mathrm{~cm}$ ) itself could have compressed the lymphatic system, hindering lymphatic drainage and resulting in decreased clearance of pleural effusion. Both a higher inflow volume into the pleural space and decreased water clearance could have been the reason for the bilateral rapid collection of pleural effusion. A similar mechanism could be considered for explaining the pulmonary edema. That is, inflow into the interstitial space was increased because of the elevated interstitial capillary pressure resulting from increased pulmonary circulation. Also, clearance of water from the interstitial space was decreased because of the elevated lymphatic pressure resulting from compression by the large DAA itself.

\section{Conclusion}

In conclusion, DAA must be borne in mind when a mass is seen in the aortic window, and fistulous DAA should be considered in the differential diagnosis of an enlarging mediastinal mass, especially when a continuous heart murmur is noted. In our patient, the cause of pulmonary edema and effusion was thought to be both elevated capillary pressure due to overload of the pulmonary circulation and decreased water clearance due to compression of the lymphatic system by the large DAA itself. DAA is a critical threat to life because of progressive compression, erosion, and hemorrhage into the esophagus or tracheobronchial tree, and rupture results in $100 \%$ mortality (7). When DAA is diagnosed, surgery should be performed without delay.

\section{References}

1) Taussig HB. Congenital Malformations of the Heart. Commonwealth Foundation, New York, 1947: 373.

2) Adachi K, Tanimura A, Nakashima T, Koga Y, Toshima H. Aneurysm of the ductus arteriosus. Angiology 29: 33-39, 1978.

3) Borow KM, Hessel SJ, Sloss LJ. Fistulous aneurysm of ductus arteriosus. Br Heart J 45: 467-470, 1981.

4) Hays JT. Spontaneous aneurysm of a patent ductus arteriosus in an elderly patient. Chest 88: 918-920, 1985.

5) Ohtsuka S, Kakihana M, Ishikawa T, et al. Aneurysm of patent ductus arteriosus in an adult case: findings of cardiac catheterization, angiography, and pathology. Clin Cardiol 10: 537-540, 1987.

6) Tofukuji M, Tabayashi K, Togo T, Ito T, Hino H, Matsuzawa K. Aneurysm of a patent ductus arteriosus in an adult. Surg Today 26: 737-739, 1996.

7) Mitchell RS, Seifert FC, Miller DC, Jamieson SW, Shumway NE. Aneurysm of the diverticulum of the ductus arteriosus in the adult. Successful surgical treatment in five patients and review of the literature. J Thorac Cardiovasc Surg 86: 400-408, 1983.

8) Lund JT, Jensen MB, Hjelms E. Aneurysm of the ductus arteriosus. A review of the literature and the surgical implications. Eur J Cardiothorac Surg 5: 566-570, 1991.

9) Cohen BA, Efremidis SC, Dan SJ, Robinson B, Rabinowitz JG. Case report. Aneurysm of the ductus arteriosus in an adult. J Comput Assist Tomogr 5: 421-423, 1981.

10) Taneja K, Gulati M, Jain M, Saxena A, Das B, Rajani M. Ductus arteriosus aneurysm in the adult: role of computed tomography in diagnosis. Clin Radiol 52: 231-234, 1997. 